\title{
Gradually varied open-channel flow profiles normalized by critical depth and analytically solved by using Gaussian hypergeometric functions
}

\author{
C.-D. Jan $^{1}$ and C.-L. Chen ${ }^{2, \dagger}$ \\ ${ }^{1}$ Department of Hydraulic and Ocean Engineering, National Cheng Kung University, Tainan 70101, Taiwan \\ ${ }^{2}$ Consulting Hydrologist, Cupertino, California, USA \\ ${ }^{\dagger}$ Deceased \\ Correspondence to: C.-D. Jan (cdjan@ mail.ncku.edu.tw)
}

Received: 18 September 2012 - Published in Hydrol. Earth Syst. Sci. Discuss.: 17 October 2012

Revised: 4 February 2013 - Accepted: 9 February 2013 - Published: 5 March 2013

\begin{abstract}
The equation of one-dimensional gradually varied flow (GVF) in sustaining and non-sustaining open channels is normalized using the critical depth, $y_{\mathrm{c}}$, and then analytically solved by the direct integration method with the use of the Gaussian hypergeometric function (GHF). The GHFbased solution so obtained from the $y_{\mathrm{c}}$-based dimensionless GVF equation is more useful and versatile than its counterpart from the GVF equation normalized by the normal depth, $y_{\mathrm{n}}$, because the GHF-based solutions of the $y_{\mathrm{c}}$-based dimensionless GVF equation for the mild (M) and adverse (A) profiles can asymptotically reduce to the $y_{\mathrm{c}}$-based dimensionless horizontal $(\mathrm{H})$ profiles as $y_{\mathrm{c}} / y_{\mathrm{n}} \rightarrow 0$. An in-depth analysis of the $y_{\mathrm{c}}$-based dimensionless profiles expressed in terms of the GHF for GVF in sustaining and adverse wide channels has been conducted to discuss the effects of $y_{\mathrm{c}} / y_{\mathrm{n}}$ and the hydraulic exponent $N$ on the profiles. This paper has laid the foundation to compute at one sweep the $y_{\mathrm{c}}$-based dimensionless GVF profiles in a series of sustaining and adverse channels, which have horizontal slopes sandwiched in between them, by using the GHF-based solutions.
\end{abstract}

\section{Introduction}

Many hydraulic engineering works involve the computation of surface profiles of gradually varied flow (GVF) that is a steady non-uniform flow in an open channel with gradual changes in its water surface elevation. The computation methods of GVF profiles in open channels have been discussed in many textbooks and journal papers (Chow, 1959;
Subramanya, 2009; Jan and Chen, 2012; Vatankhah, 2012). The most widely used methods for computing GVF profiles could be classified into the step methods and the direct integration methods. The step methods are numerical methods and are primarily used in natural channels with non-prismatic sections. On the other hand, the direct integration methods involve the integration of the GVF equation and may be performed by using analytical, semi-analytical, or numerical procedures. Numerical integration of the GVF equation is primarily used in non-prismatic channels. In some prismatic channels, such as artificial channels, the GVF equation can be simplified so as to let the analytical (or semi-analytical) direct integration be applied. The analytical direct-integration method is straightforward and can provide the total length of the profile in a single computation step. In the directintegration method, the one-dimensional (1-D) GVF equation is usually normalized to be a simpler expression in advance so as to allow the performance of direct integration. In most cases, the GVF equation is normalized by the normal depth $y_{\mathrm{n}}$ (Chow, 1959; Subramanya, 2009; Jan and Chen, 2012; Venutelli, 2004; Vatankhah, 2012), while in some cases, it is normalized by the critical depth $y_{\mathrm{c}}$ (Chen and Wang, 1969). Many attempts have been made by previous investigators on the direct-integration method. The varied-flow function (VFF) needed in the direct-integration method conventionally used by Bakhmeteff (1932), Chow $(1955,1957$, 1959), Kumar (1978), among others, has a drawback caused by the imprecise interpolation of the VFF values. To overcome the drawback, Jan and Chen (2012) already successfully used the Gaussian hypergeometric functions (GHFs) 
to analytically solve the GVF equation in sustaining wide channels without recourse to the VFF.

Success to formulate the normal-depth $\left(y_{n}\right)$-based nondimensional GVF profiles expressed in terms of GHFs for flow in sustaining channels, as reported by Jan and Chen (2012), does not necessarily warrant that it can likewise prevail to use $y_{\mathrm{n}}$ in the normalization of the GVF equation for flow in adverse channels because $y_{\mathrm{n}}$ for an assumed uniform flow in adverse channels is undefined. Even though one can use an imaginary flow resistance coefficient to evaluate $y_{\mathrm{n}}$ for such an assumed uniform flow in adverse channels (Chow, 1959), as will be treated later in this paper, it is inappropriate to use such an $y_{\mathrm{n}}$ in the normalization of the GVF equation for flow in adverse channels because $y_{\mathrm{n}}$ so evaluated is fictitious and the two normalized variables in such $y_{\mathrm{n}}$-based dimensionless GVF equation collapse (in virtue of $y_{n} \rightarrow \infty$ ) as the channel-bottom slope approaches zero. To fill such a mathematical gap as a result of the indiscreet choice of a characteristic length in the normalization of the GVF equation for adverse channels, Chen and Wang (1969) used $y_{\mathrm{c}}$ to replace $y_{\mathrm{n}}$ along to derive two $y_{\mathrm{c}}$-based dimensionless GVF equations, one for sustaining channels and the other for adverse channels. For the hydraulic exponents $M=3$ (wide channels) and $N=10 / 3$ (equivalent to using Manning's formula) they integrated both equations numerically over the normalized depth, $y / y_{\mathrm{c}}$, to get the $y_{\mathrm{c}}$-based dimensionless GVF profiles in sustaining and adverse channels.

This study focuses on the direct integration method that used to analytically compute the GVF profiles in sustaining and non-sustaining channels, in which the GVF equation is normalized by using $y_{\mathrm{c}}$ and then analytically solved by using the GHFs. Before proceeding to formulate the two $y_{\mathrm{c}}$-based dimensionless GVF equations for sustaining channels and adverse channels, respectively, it is worthwhile to review all of what has been attained by previous investigators to compute the GVF profiles in both sustaining and adverse channels. We already reviewed quite comprehensively most of the previous work on the analytical computation of the GVF profiles in sustaining channels, as reported in our paper (Jan and Chen, 2012). Therefore, in this paper, we focus on the literature survey only of the computed GVF profiles in adverse channels.

As a matter of fact, there are not many previous investigators who have contributed to the computation of the GVF profiles in adverse channels. One of the earliest investigations in this study may be credited to Matzke (1937), who formulated the $y_{\mathrm{n}}$-based dimensionless GVF equation for GVF in adverse channels, thereby integrating the indefinite integrals of the GVF equation in the form of the variedflow function (VFF), following in Bakhmeteff's (1932) footsteps. However, unlike the four methods used by Bakhmeteff to compute the VFF values numerically, Matzke evaluated them by a graphical method and constructed a table thereof for any $N$ values between 3 and 4, inclusive, for computing the $y_{\mathrm{n}}$-based dimensionless GVF profiles in adverse chan- nels. Chow $(1957,1959)$ was probably the first person who directly computed the VFF values for GVF in adverse channels from the corresponding two transformed VFF values for GVF in sustaining channels. He further tabulated the VFF values so computed for $N$ values ranging from 3 to 5.5 for use in engineering applications to compute the $y_{\mathrm{n}}$-based dimensionless GVF profiles in adverse channels.

In the direct integration of the GVF equation, regardless of whether it is carried out for sustaining or adverse channels, most previous investigators expressed the rational function representing the reciprocal of the slope of the GVF profile in terms of the dimensionless flow depth rather than in terms of the geometric elements of a given channel section before the two indefinite integrals were evaluated. Nevertheless, Allen and Enever (1968) as well as Kumar (1979) did it in reverse by substituting their geometric elements of a rectangular, trapezoidal, or triangular channel section into the section factor, $Z$, and the conveyance, $K$, of the channel section in the GVF equation before evaluating the two indefinite integrals. Subsequently, they evaluated the two indefinite integrals using the partial-fraction expansion and then got the elementary-transcendental-function (ETF)-based solution for each of the cross-sectional shapes under study, such as rectangular, triangular and parabolic cross-sectional shapes. Another approach taken by Zaghloul $(1990,1992)$ to integrate the GVF equation for the profiles in circular pipes was the same as that used by Allen and Enever (1968) by substituting the geometric elements of a circular conduit section into $Z$ and $K$ in the GVF equation before integrating it by use of Simpson's rule (Zaghloul, 1990) or both the direct step and integration methods (Zaghloul, 1992). After all, he developed a computer model on the basis of such methods to compute GVF profiles not only in sustaining and horizontal pipes, but also in adverse pipes.

For computing GVF profiles in sustaining channels, one can solve the $y_{\mathrm{n}}$-based dimensionless GVF equation by using the direct integration method and the Gaussian hypergeometric function (GHF), as presented by Jan and Chen (2012). Nevertheless, for computing GVF profiles in adverse channels, one cannot do likewise due to the difficulty in quantifying the $y_{\mathrm{n}}$-value to be used in the normalization of the GVF equation because $y_{\mathrm{n}}$ for an assumed uniform flow in adverse channels is undefined. Use of $y_{\mathrm{c}}$ instead of $y_{\mathrm{n}}$ in the normalization of the GVF equation for flow in adverse channels can resolve such a vital issue resulting from the undefined $y_{\mathrm{n}}$ because $y_{\mathrm{c}}$ can be uniquely determined for a given discharge. The primary objective of this paper is thus to formulate and then analytically solve the two $y_{\mathrm{c}}$-based dimensionless GVF equations for GVFs in both sustaining and adverse channels by using the direct integration method and the Gaussian hypergeometric functions (GHFs). The GHFbased solutions of this study will lay the theoretical foundation to compute such $y_{\mathrm{c}}$-based dimensionless GVF profiles in a series of interconnected sustaining and adverse channels. 


\section{Critical-depth-based dimensionless GVF equations}

Before deriving the $y_{\mathrm{c}}$-based dimensionless one dimensional GVF profiles in sustaining channels $\left(S_{0}>0\right)$, horizontal channels $\left(S_{0}=0\right)$, and adverse channels $\left(S_{0}<0\right)$, we first formulate the $y_{\mathrm{c}}$-based dimensionless GVF equations for three such types of channels in the following.

\subsection{Equation of the GVF in sustaining channels}

The dimensional form of the 1-D GVF equation expressed in terms of the flow depth prior to normalization can be written as (Chow, 1959)

$$
\frac{\mathrm{d} y}{\mathrm{~d} x}=S_{0} \frac{1-\left(y_{\mathrm{n}} / y\right)^{N}}{1-\left(y_{\mathrm{c}} / y\right)^{M}} .
$$

This equation is a first order differential equation and denotes the relation between the flow depth $y$ and the axial distance $x$ along the open channel having a slope $S_{0}$. The subscripts " $\mathrm{n}$ " and "c" refer to the normal and critical flow conditions, respectively. The exponents $M$ and $N$ are usually called the hydraulic exponents for critical-flow computation and uniformflow computation, respectively. It is expected that the solution of Eq. (1), if obtainable, can be expressed in a form of $y$ as an implicit function of $x$ with $S_{0}, y_{\mathrm{n}}, y_{\mathrm{c}}, M$, and $N$ as parameters.

Though the validity of Eq. (1) is not at issue, one should be aware of its implication before normalizing it for solution. Firstly, the resistance to GVF in open channels, as incorporated in Eq. (1), is conventionally evaluated on the assumption that the resistance at any section is equal to what it would be as if the same $Q$ passed through the same section under conditions of uniformity. However, this conventional assumption made in Eq. (1) does not take the effect of boundary non-uniformity into account in the evaluation of the non-uniform flow resistance. According to Rouse (1965), the effect of boundary non-uniformity on the flow resistance should include any change in shape or size of the section along the longitudinal direction. Secondly, Eq. (1) cannot be used to compute GVF profiles in nonsustaining (horizontal or adverse) channels because $y_{\mathrm{n}}$ is infinite for flow in horizontal channels and undefined for flow in adverse channels. Thirdly, $N$ is related to the power in the power-law flow resistance formula, which has been incorporated in formulating Eq. (1). It is noted that $N=3$ for hydraulically smooth flows and $N=2 m+3$ (where $m$ is the exponent of a unified similarity variable used in the power laws of the wall) for fully rough flows (Chen, 1991).

By and large, there are two ways in which $x$ and $y$ in Eq. (1) can be normalized: one is based on the normal depth, $y_{\mathrm{n}}$, as usually adopted by many researchers (Chow, 1959) for GVF in sustaining channels, and the other based on the critical depth, $y_{\mathrm{c}}$, as treated by Chen and Wang (1969), who adopted $y_{\mathrm{c}}$ in place of $y_{\mathrm{n}}$ to compute in one sweep GVF profiles in a series of interconnected sustaining and nonsustain- ing channels. Therefore, for the first case in which Eq. (1) is normalized based on $y_{\mathrm{n}}$, we can introduce the dimensionless variables $u\left(=y / y_{\mathrm{n}}\right)$ and $x_{*}\left(=x S_{0} / y_{\mathrm{n}}\right)$, as done by Chow (1959), Jan and Chen (2012) among others, thereby expressing the reciprocal of Eq. (1) in terms of $u$ and $x_{*}$ as

$\frac{\mathrm{d} x_{*}}{\mathrm{~d} u}=\frac{-u^{N}+\lambda^{M} u^{N-M}}{1-u^{N}}$

which has been referred to as the dimensionless GVF equation based on $y_{\mathrm{n}}$ with $\lambda\left(=y_{\mathrm{c}} / y_{\mathrm{n}}\right), M$, and $N$ as three parameters. The characteristic length ratio $\lambda$ in Eq. (2) is the primary parameter that classifies the GVF profiles into those in the mild $(\mathrm{M})$, critical $(\mathrm{C})$, and steep $(\mathrm{S})$ channels if the ratio $\lambda$ is less than, equal to, and larger than unity, respectively.

Instead of $y_{\mathrm{n}}$, we want to use $y_{\mathrm{c}}$ to normalize $x$ and $y$ in Eq. (1) herein by simply adopting the dimensionless variables $v\left(=y / y_{\mathrm{c}}\right)$ and $x_{\#}\left(=x S_{\mathrm{c}} / y_{\mathrm{c}}\right)$, as done by Chen and Wang (1969), to rewrite the reciprocal of Eq. (1) in terms of $v$ and $x_{\#}$ so as to obtain the following $y_{\mathrm{c}}$-based dimensionless GVF equation with $\lambda, M$, and $N$ as three parameters.

$\frac{\mathrm{d} x_{\#}}{\mathrm{~d} v}=\frac{-v^{N}+v^{N-M}}{1-(\lambda v)^{N}}$

which is valid in the domain of $0 \leq v<\infty$.

The following two relations are the normalized flow depth and normalized longitudinal coordinate defined on the basis of $y_{\mathrm{n}}$ to their counterparts defined on the basis of $y_{\mathrm{c}}$ :

$$
\begin{aligned}
& \frac{u}{v}=\frac{y_{\mathrm{c}}}{y_{\mathrm{n}}} \\
& \frac{x_{*}}{x_{\#}}=\frac{y_{\mathrm{c}}}{y_{\mathrm{n}}} \frac{S_{0}}{S_{\mathrm{c}}}=\left(\frac{y_{\mathrm{c}}}{y_{\mathrm{n}}}\right)^{N+1} .
\end{aligned}
$$

Both normalized variables so related in Eqs. (4) and (5) will be useful in converting the solutions of Eq. (2) to its counterparts obtained from Eq. (3), or vice versa.

\subsection{Equation of the GVF in horizontal channels}

Although Chow (1959, p. 223) did not classify horizontal channels as a type of sustaining channels, we can prove that the $y_{\mathrm{c}}$-based dimensionless GVF equation, Eq. (3), is still valid for GVF profiles in horizontal channels. In other words, because we have $S_{0}=0, y_{\mathrm{n}} \rightarrow \infty$, and $\lambda\left(=y_{\mathrm{c}} / y_{\mathrm{n}}\right) \rightarrow$ 0 for GVF profiles in horizontal channels, Eq. (3) can be simplified to

$\frac{\mathrm{d} x_{\#}}{\mathrm{~d} v}=v^{N-M}-v^{N}$

which is still valid in the domain of $0 \leq v<\infty$. Equation (6) is the dimensionless equation based on $y_{\mathrm{c}}$ for GVF profiles in horizontal channels with $M$ and $N$ as two parameters. For convenience, Eq. (6) may be referred to as the asymptote of Eq. (3) as $\lambda \rightarrow 0$. Under the assumption of constant $M$ and 
$N$, Eq. (3) can be analytically integrated over $v$ using the $\mathrm{GHF}$, as will be shown later, while we can directly integrate Eq. (6) without resorting to the GHF in the following manner.

$x_{\#}=\frac{v^{N-M+1}}{N-M+1}-\frac{v^{N+1}}{N+1}+$ Const

The above equation describes the $y_{\mathrm{c}}$-based dimensionless GVF profiles in wide horizontal channels (in which $M=3$ ). The constant of integration, "Const", in Eq. (7) is to be determined from a given boundary condition. It should be noted that Eq. (7) can readily be identified with Chow's (1959, Sect. 10.2) Eqs. (10)-(26).

Chow's (1959, Sect. 10.2) classification to distinguish a horizontal slope $\left(S_{0}=0\right)$ from a sustaining slope $\left(S_{0}>0\right)$ or an adverse slope $\left(S_{0}<0\right)$ seems at odds with the limit of differential calculus, because the $y_{\mathrm{c}}$-based dimensionless horizontal profiles, Eq. (7), can be derived from the $y_{\mathrm{c}}$-based dimensionless equation for GVF profiles in sustaining or adverse channels having any real $S_{0}$-values. In fact, Chow could have treated GVF profiles on a horizontal slope as the asymptote of those on a sustaining slope as $S_{0} \rightarrow 0$, as deduced above from Eqs. (3) to (7) by letting $\lambda \rightarrow 0$ (or $y_{\mathrm{n}} \rightarrow \infty$ ). We shall also later prove that the GHF-based solutions of Eq. (3) for GVF on the mild slope (where $0<\lambda<1$ ) can reduce to Eq. (7) when we approach its asymptote by letting $\lambda \rightarrow 0$. Likewise, the $y_{\mathrm{c}}$-based dimensionless equation for GVF in adverse channels, as will be formulated later, can also approach its asymptote, namely Eq. (7), as $\lambda \rightarrow 0$. Therefore, for coherence in treating the asymptote of the $y_{\mathrm{c}}$-based dimensionless GVF equation and its solutions throughout the paper, we henceforth regard the horizontal slope as an intermediate (or interface) between the sustaining slope and the adverse slope as $S_{0}$ approaches zero from either slope.

\subsection{Equation of the GVF in adverse channels}

To formulate the $y_{\mathrm{c}}$-based dimensionless GVF equation for GVF in adverse channels, we deem the slope of the channel bottom as negative, i.e. $S_{0}<0$. Such a fictitious treatment may result in an imaginary value of $K_{\mathrm{n}}$ (the conveyance for uniform flow at a depth $y_{\mathrm{n}}$ ) or a negative value of $K_{\mathrm{n}}^{2}$ for a given $Q\left(=K_{\mathrm{n}} S_{0}^{q}\right.$, provided $\left.q=1 / 2\right)$. Although $y_{\mathrm{n}}$ for uniform flow in adverse channels is undefined, we may assume that the coefficient of the flow resistance, $C$, in the flow resistance formula is imaginary so as to enable the computation of $y_{\mathrm{n}}$. In other words, for a negative value of $K_{\mathrm{n}}^{2}$, the value of $C^{2}$ used in computing $y_{\mathrm{n}}$ from $K_{\mathrm{n}}^{2}=C^{2} A_{\mathrm{n}}^{2} R_{\mathrm{n}}^{2 p}$ (where $p$ is the exponent related to the flow resistance formula; $p=2 / 3$ for Manning's formula and $p=1 / 2$ for Chézy formula) must be negative, thus resulting in a fictitious value of $y_{n}$, if determined from the above relation. Because the critical depth $y_{\mathrm{c}}$ can be determined from the critical relation for a given $Q$ (Chow, 1959, Sect. 4.2), the fictitious $y_{\mathrm{n}}$ so calculated can be further incorporated with $y_{\mathrm{c}}$ to derive the following $y_{\mathrm{c}}$-based dimensionless equation for GVF in adverse channels.

$\frac{\mathrm{d} x_{\#}}{\mathrm{~d} v}=\frac{-v^{N}+v^{N-M}}{1+(\lambda v)^{N}}$

in which $v=y / y_{\mathrm{c}}$ and $x_{\#}=x S_{\mathrm{c}} / y_{\mathrm{c}}$, the same dimensionless variables used in the derivation of Eq. (3). As pointed out earlier, Eq. (8) for GVF in adverse channels can reduce asymptotically to Eq. (6) for GVF in horizontal channels as $S_{0} \rightarrow 0$ (i.e. $y_{\mathrm{n}} \rightarrow \infty$ or $\lambda \rightarrow 0$ ).

Undoubtedly, at issue herein is the controversial approach taken in evaluating $y_{\mathrm{n}}$ for use in Eq. (8) because the resistance coefficient in the flow resistance formula is assumed to be imaginary and the $y_{n}$-value so evaluated is fictitious in an adverse channel. Nevertheless, for a given $Q$, the $y_{\mathrm{c}}$-value so computed is unique, regardless of whether it is in a sustaining or adverse channel. The parameter, $\lambda^{N}$, appearing in Eq. (8) signifies the slope ratio, $S_{\mathrm{o}} / S_{\mathrm{c}}$, thus implying that $\lambda^{N}$ is of a negative value for GVF in adverse $\left(S_{0}<0\right)$ channels. Therefore, $\lambda\left(=\left(S_{0} / S_{\mathrm{c}}\right)^{1 / N}\right)$ as a parameter for GVF in adverse channels is not so physically meaningful as that in Eq. (3) for GVF in sustaining channels, in which the flow criteria of the $\lambda$-value being less than, equal to, and greater than unity can represent respectively the GVF on the mild, critical, and steep slopes. In contrast, the fictitious $\lambda$-value in Eq. (8) for GVF in adverse channels is nothing but an index. Although the ultimate usefulness of Eq. (8) may not be realized until after such an issue in the evaluation of $y_{\mathrm{n}}$ is resolved, we presently rest content with such fictitious $y_{\mathrm{n}}$ because there appears no other approach better than that taken above to evaluate $y_{\mathrm{n}}$ for GVF in adverse channels.

Although $y_{n}$-value so evaluated is fictitious in an adverse channel regardless of whether Eq. (1) is normalized on the basis of $y_{\mathrm{c}}$ or $y_{\mathrm{n}}$, we prefer to normalize Eq. (1) based on $y_{\mathrm{c}}$ rather than based on $y_{\mathrm{n}}$ for the following main reason. Most previous investigators, such as Chow $(1957,1959)$ formulated the $y_{\mathrm{n}}$-based dimensionless GVF equation for computing GVF profiles in adverse channels, i.e., the $y_{\mathrm{n}}$-based counterpart of Eq. (8) instead of the $y_{c}$-based Eq. (8). However, Eq. (8) has the obvious advantage over its $y_{n}$-based counterpart because the latter cannot reduce asymptotically to Eq. (6) as $S_{0} \rightarrow 0$ (i.e. $y_{\mathrm{n}} \rightarrow \infty$ or $\lambda \rightarrow 0$ ) unless the normalizing quantity of the $y_{\mathrm{n}}$-based GVF equation for adverse channels can be altered from $y_{\mathrm{n}}$ to $y_{\mathrm{c}}$ so as to enable one to switch the normalized variables from $u$ to $v$ via Eq. (4) and from $x_{*}$ to $x_{\#}$ via Eq. (5) as $S_{0} \rightarrow 0$. By implication, if the $y_{\mathrm{n}}$-based counterpart of Eq. (8) would have been used to compute GVF profiles in adverse channels, the horizontal slope could not have been treated as an intermediate (or interface) between the sustaining slope and the adverse slope as $S_{0} \rightarrow 0$. Insomuch that, we can deem Eq. (8) with the parameter, $\lambda$, a completely general equation to compute the $y_{\mathrm{c}^{-}}$ based dimensionless GVF profiles on adverse slopes, especially versatile in a series of interconnected sustaining and adverse channels, having horizontal channels sandwiched in between them. 


\section{Gaussian hypergeometric function}

Prior to find the analytical solutions of the critical-depthbased equations for the gradually varied flows in sustaining and adverse channels by using the Gaussian hypergeometric function (GHF), we will introduce the definition of GHF and some relations first. GHF can be expressed as an infinite series and symbolized in the form of ${ }_{2} F_{1}(a, b ; c ; z)$ as shown in the books of Korn and Korn (1961), Luke (1975), Pearson (1974), and Seaborn (1991). The form ${ }_{2} F_{1}(a, b ; c ; z)$ can be safely changed to the more friendly form $F(a, b ; c$; $z)$ as Olde Daalhuis (2010, Sect. 15.1) indicates, i.e.

$$
F(a, b ; c ; z)=\frac{\Gamma(c)}{\Gamma(a) \Gamma(b)} \sum_{s=0}^{\infty} \frac{\Gamma(a+s) \Gamma(b+s)}{\Gamma(c+s) s !} z^{s}
$$

in which $a, b$, and $c$ are the function parameters and $z$ is the variable. The infinite series in Eq. (9) is convergent for arbitrary $a, b$, and $c$, provided that $c$ is neither a negative integer nor zero and that $a$ or $b$ is not a negative integer for real $-1<z<1$ (or $|z|<1$ ), and for $z= \pm 1$ if $c>a+b$ (Olde Daalhuis, 2010). Equation (9) has the symmetric property: $F(a, b ; c ; z)=F(b, a ; c ; z)$.

A survey of the literature reveals that there are two linearly independent solutions of the hypergeometric differential equation at each of the three singular points $z=0,1$, and $\infty$ for a total of six special solutions, which are in fact fundamental to Kummer's (1836) 24 solutions. Any three of the aforementioned six special solutions satisfy a relation, thus giving rise to twenty combinations thereof. Among them, there is a relation connecting one GHF in the domain of $|z|<1$ to two GHFs in the domain of $|z|>1$ in the following way (Luke, 1975):

$$
\begin{aligned}
& F(a, b ; c ; z)=\frac{\Gamma(b-a) \Gamma(c)}{\Gamma(b) \Gamma(c-a)}\left(-z^{-1}\right)^{a} \\
& F\left(a, a-c+1 ; a-b+1 ; z^{-1}\right)+\frac{\Gamma(a-b) \Gamma(c)}{\Gamma(a) \Gamma(c-b)}\left(-z^{-1}\right)^{b} \\
& F\left(b, b-c+1 ;-a+b+1 ; z^{-1}\right)
\end{aligned}
$$

One may use Eq. (10) to transform the GHF-based solutions in the domain of $|z|<1$ to their counterparts in the domain of $|z|>1$. The commercial Mathematica software treats Eq. (10) as a definition of $F(a, b ; c ; z)$ for $|z|>1$ (Wolfram, 1996).

For the special case, $a, b$, and $c$ are fixed with specified relations, $a=1$ and $c=b+1$, Eq. (9) can be written as

$F(1, b ; b+1 ; z)=g(b, z)=b \sum_{s=0}^{\infty} \frac{z^{s}}{b+s}$.

The GHFs used in the solutions of the gradually varied flow profiles herein are all in the form of Eq. (11). Since the first argument of the GHF of Eq. (11) is always unity, the second and third arguments differ in one unity, and the fourth argument is a variable only, we can express the GHF in a simpler expression, such as $g(b, z)=F(1, b ; b+1 ; z)$, to reduce the related equations to shorter expressions for facilitating the reading of the manuscript, as treated by Jan and Chen (2012). Therefore, we will use $g(b, z)$ instead of $F(1, b ; b+1 ; z)$ herein to represent GHF when we treat the GHF-solutions in the present paper.

The following recurrence formulas between two GHFs can be generally established from Eq. (11) as shown in Jan and Chen (2012).

$g(b, z)=1+\frac{b z}{b+1} g(b+1, z)$.

Since $\Gamma(b)=(b-1) \Gamma(b-1), \Gamma(b+1)=b \Gamma(b)$, and $F$ $\left(b, 0 ; b ; z^{-1}\right)=1$, we can rewrite Eq. (10), for $|z|>1$, in a much shorter expression as

$$
\begin{aligned}
& g(b, z)=-\frac{b z^{-1}}{b-1} g\left(1-b, z^{-1}\right) \\
& . \Gamma(1-b) \Gamma(1+b)\left(-z^{-1}\right)^{b}
\end{aligned}
$$

In addition, for the condition $(\beta t)^{N}<1$ (i.e. $\beta t<1$ ), in which $\beta$ is a positive parameter, $t$ is a positive variable, and $N$ is a positive real number, using the commercial Mathematica software (Wolfram, 1996), we can find the following general relation for the solution of the indefinite integral in terms of GHF as shown below.

$$
\int \frac{t^{\phi}}{1 \mp(\beta t)^{N}} d t=\frac{t^{\phi+1}}{\phi+1} g\left(\frac{\phi+1}{N}, \pm(\beta t)^{N}\right)+\text { Const }
$$

in which the parameter $\phi$ is a real number. The term $(\beta t)$ in Eq. (14) could be $(\lambda v)$ or $(w / \lambda)$ for the study herein as shown in the next sections. This kind of indefinite integrals as shown in Eq. (14) will be applied many times in the following sections when we find GHF-based solutions for GVF profiles in sustaining and adverse channels.

\section{Analytical solutions of the $y_{c}$-based GVF equations}

There are two ways to analytically solve the normal-depthbased ( $y_{\mathrm{n}}$-based) dimensionless GVF equation by the directintegration method: One is based on the GHF and the other on the elementary transcendental functions (ETF). The innovative results obtained by Jan and Chen (2012) shows that the GHF-based solutions are more useful and versatile than the ETF-based solutions, because the former allows its involved parameters $M$ and $N$ being real numbers while the latter can at the most accept rational numbers, but not real numbers. This paper is henceforth focused only on the acquisition and analysis of the GHF-based solutions for the critical-depthbased ( $y_{\mathrm{c}}$-based) dimensionless GVF equation. Analogous to the direct integration of the $y_{\mathrm{n}}$-based dimensionless GVF equation, as treated by Jan and Chen (2012), we can directly integrate both Eqs. (3) and (8) for the sustaining and adverse channels respectively, and then express their solutions in terms of the GHF by using Eq. (14). 


\subsection{Alternative forms of Eqs. (3) and (8)}

The validity of the solutions of Eq. (3) or (8) to be expressed in terms of the GHF is confined to the convergence criterion of GHF itself, i.e. $|\lambda v|<1$. Therefore, an alternative form of Eq. (3) or (8) has to be formulated so as to allow its solutions expressed in terms of the GHF be valid for $|\lambda v|>1$. Every GHF-based solution of Eq. (3) or (8) thus consists of two parts: one in the domain of $|\lambda v|<1$ and the other in the domain of $|\lambda v|>1$. To derive alternative forms of Eqs. (3) and (8) in order that their GHF-based solutions be valid for $|\lambda v|>1$, Eqs. (3) and (8) on substitution of $v=w^{-1}$ and $\mathrm{d} v$ $=-w^{-2} \mathrm{~d} w$ will respectively yield the following Eqs. (15) and (16) for GVF in sustaining and adverse channels, respectively.

$$
\begin{aligned}
& \frac{\mathrm{d} x_{\#}}{\mathrm{~d} w}=\lambda^{-N} \frac{-w^{-2}+w^{M-2}}{1-(w / \lambda)^{N}} \\
& \frac{\mathrm{d} x_{\#}}{\mathrm{~d} w}=\lambda^{-N} \frac{w^{-2}-w^{M-2}}{1+(w / \lambda)^{N}}
\end{aligned}
$$

Before procuring the GHF-based solutions from Eqs. (15) and (16), it merits mentioning that such GHF-based solutions, if obtainable, are premised on the assumption that they are valid only in the domains of $|w / \lambda|<1$ (i.e. $|\lambda v|>1$ ).

\subsection{GVF profiles in sustaining channels}

For facilitating the direct integration to obtain the GHF-based solutions of Eq. (3) for $|\lambda v|<1$ and Eq. (14) for $|\lambda v|>1$, we can rearrange Eq. (3) into three different forms. The righthand side of Eq. (3) is the rational function of $v$, which by the process of long division can be expressed in the form of a polynomial plus a proper fraction in two ways before we integrate them. One way is to divide the first term of the numerator on the right-hand side of Eq. (3) by the denominator; the other way is to divide the second term of the numerator on the right-hand side of Eq. (3) by the denominator. Therefore, they are three possible representations of the solution of Eq. (3) by the direct integration method, as shown below.

$$
\begin{aligned}
x_{\#} & =-\int \frac{v^{N}}{1-(\lambda v)^{N}} \mathrm{~d} v+\int \frac{v^{N-M}}{1-(\lambda v)^{N}} \mathrm{~d} v+\text { Const } \\
x_{\#}= & \frac{v^{N-M+1}}{N-M+1}-\int \frac{v^{N}}{1-(\lambda v)^{N}} \mathrm{~d} v+\lambda^{N} \\
& \int \frac{v^{2 N-M}}{1-(\lambda v)^{N}} \mathrm{~d} v+\text { Const } \\
x_{\#}= & -\frac{v^{N+1}}{N+1}+\int \frac{v^{N-M}}{1-(\lambda v)^{N}} \mathrm{~d} v-\lambda^{N} \\
& \int \frac{v^{2 N}}{1-(\lambda v)^{N}} \mathrm{~d} v+\text { Const }
\end{aligned}
$$

The GHF-based solutions for Eqs. (17), (18) and (19) can be easily obtained by using the help of Eq. (14). Three GHFbased solutions to be obtained from Eq. (17) to Eq. (19) should be identical. Equating any two of the three GHFbased solutions will thus establish a recurrence formula between the two contiguous GHFs as shown in Eq. (12). Only one of the three solutions is needed. Because Eq. (17) has shorter length of expression, we will use it latter to derive our GHF-based solutions for GVF profile in a sustaining channel.

Likewise, to derive the GHF-based solutions of Eq. (15) for $|\lambda v|>1$, we can make a direct integration to Eq. (15) as shown below.

$$
\begin{gathered}
x_{\#}=-\lambda^{-N} \int \frac{w^{-2}}{1-(w / \lambda)^{N}} \mathrm{~d} w+\lambda^{-N} \\
\int \frac{w^{M-2}}{1-(w / \lambda)^{N}} \mathrm{~d} w+\text { Const }
\end{gathered}
$$

With the help of Eq. (14), the complete GHF-based solutions of the dimensionless GVF equation, Eq. (3), for GVF in sustaining channels can be obtained by executing the integration of the two indefinite integrals in Eq. (17) for $|\lambda v|<1$ and then carrying out the same in Eq. (20) for $|w / \lambda|<1$ (or $|\lambda v|>1)$, as shown below.

$$
\begin{aligned}
x_{\#}= & -\frac{v^{N+1}}{N+1} g\left(\frac{N+1}{N},(\lambda v)^{N}\right)+\frac{v^{N-M+1}}{N-M+1} g \\
& \left(\frac{N-M+1}{N},(\lambda v)^{N}\right)+\text { Const }
\end{aligned}
$$

which is valid only for $|\lambda v|<1$.

$$
\begin{gathered}
x_{\#}=\lambda^{-N} w^{-1} g\left(\frac{-1}{N},\left(\frac{w}{\lambda}\right)^{N}\right)+\frac{\lambda^{-N} w^{M-1}}{M-1} g \\
\left(\frac{M-1}{N},\left(\frac{w}{\lambda}\right)^{N}\right)+\text { Const }
\end{gathered}
$$

which is valid only for $|w / \lambda|<1$. To express Eq. (22) in terms of $v$, we substitute $w=v^{-1}$ into Eq. (22) to have

$$
\begin{aligned}
x_{\#}= & \lambda^{-N} v g\left(-\frac{1}{N},(\lambda v)^{-N}\right)+\frac{\lambda^{-N} v^{-M+1}}{M-1} g \\
& \left(\frac{M-1}{N},(\lambda v)^{-N}\right)+\text { Const }
\end{aligned}
$$

which is valid only for $|\lambda v|>1$.

It is worthwhile to reiterate that we can use Eq. (13) to transform the GHF-based solutions in the domain of $|z|<1$ to their counterparts in the domain of $|z|>1$. Here $z=(\lambda v)^{N}$ for Eq. (21). That is to say that we can use Eq. (13) to directly transform Eq. (21) to Eq. (23), vice versa, without recourse to the formulation of Eq. (15) [i.e. an alternative form of Eq. (3) with its variable, $w$, being expressed as $\left.v^{-1}\right]$, which can be solved for GVF profiles in the domains of $|w / \lambda|<1$ (i.e. $|\lambda v|>1$ ), thereby proving that we can obtain Eq. (23) directly from Eq. (21) rather than indirectly through Eq. (15). 
It should be noted that such GHF-based solutions exclude points where $|\lambda v|=1$ (or $|w / \lambda|=1$ ) because the GHF diverges at $|\lambda v|=1$ (or $|w / \lambda|=1$ ). Physically, such points correspond to the convergent limits of the GHF in the GHF-based solutions of Eq. (3) at $x_{\#}=\infty$, where GVF profiles run parallel with the channel bottom.

Although the absolute value of the variable in the GHF, i.e. $|(\lambda v)|$, has been imposed to derive the GHF-based solutions of Eq. (3) for GVF profiles in sustaining channels, the complete GHF-based solutions of Eq. (3) can only cover the physically possible domain of $\lambda v$, i.e. $0 \leq \lambda v<\infty$, thus consisting of Eq. (21) for $0 \leq \lambda v<1, x_{\#}=\infty$ at $\lambda v=1$, and Eq. (23) for $\lambda v>1$. Insomuch that we can lift the absolutevalue restriction imposed on $\lambda v$ in expressing the GHF-based solutions without the loss of generality.

\subsection{GVF profiles in adverse channels}

By the same token, the solution of the GVF profiles in adverse channels by the direct integration method can be obtained from Eq. (8) for $|\lambda v|<1$ and Eq. (16) for $|\lambda w|<1$ (i.e. $|\lambda v|>1$ ), as shown below.

$$
\begin{gathered}
x_{\#}=-\int \frac{v^{N}}{1+(\lambda v)^{N}} \mathrm{~d} v+\int \frac{v^{N-M}}{1+(\lambda v)^{N}} \mathrm{~d} v+\text { Const } \\
x_{\#}=\lambda^{-N} \int \frac{w^{-2}}{1+(w / \lambda)^{N}} \mathrm{~d} w-\lambda^{-N} \\
\int \frac{w^{M-2}}{1+(w / \lambda)^{N}} \mathrm{~d} w+\text { Const }
\end{gathered}
$$

With the help of Eq. (14), the complete GHF-based solutions of Eq. (8) for dimensionless GVF profiles in adverse channels can be obtained by executing the integration of the two indefinite integrals in Eq. (24) for $|\lambda v|<1$ and then carrying out the same in Eq. (25) for $|w / \lambda|<1$ (or $|\lambda v|>1$ ). The results are

$$
\begin{gathered}
x_{\#}=-\frac{v^{N+1}}{N+1} g\left(\frac{N+1}{N},-(\lambda v)^{N}\right)+\frac{v^{N-M+1}}{N-M+1} g \\
\left(\frac{N-M+1}{N},-(\lambda v)^{N}\right)+\text { Const } \\
x_{\#}=-\lambda^{-N} w^{-1} g\left(-\frac{1}{N},-\left(\frac{w}{\lambda}\right)^{N}\right)-\frac{\lambda^{-N} w^{M-1}}{M-1} g \\
\left(\frac{M-1}{N},-\left(\frac{w}{\lambda}\right)^{N}\right)+\text { Const. }
\end{gathered}
$$

Equations (26) and (27) should be valid in the domain of $|\lambda v|<1$ and $|w / \lambda|<1$, respectively. To express Eq. (27) in terms of $v$, substituting $w=v^{-1}$ into Eq. (27) yields

$$
\begin{gathered}
x_{\#}=-\lambda^{-N} v g\left(-\frac{1}{N},-(\lambda v)^{-N}\right)-\frac{\lambda^{-N} v^{-M+1}}{M-1} g \\
\left(\frac{M-1}{N},-(\lambda v)^{-N}\right)+\text { Const }
\end{gathered}
$$

which is valid only for $|\lambda v|>1$.

It is also found that the two GHFs appearing in each of Eqs. (26) and (28) converge but not diverge at $\lambda v=1$ because the variable of both GHFs is of a negative value through its entire domain of $\lambda v \geq 0$. That is to say that Eqs. (26) and (28) cover the domains of $0 \leq \lambda v \leq 1$ and $\lambda v \geq 1$, respectively. Although the absolute value of the variable in the GHF, i.e. $|\lambda v|$, has been imposed to derive the GHF-based solutions from both Eqs. (8) and (16), the complete GHF-based solutions of Eq. (8) for GVF profiles in adverse channels can only cover the physically possible domain of $\lambda v \geq 0$. It is thus inferred that the A1 profile does not exist.

It is worthwhile to reiterate that we can use Eq. (13) to transform the GHF-based solutions in the domain of $|z|<1$ to their counterparts in the domain of $|z|>1$. Here $z=-(\lambda v)^{N}$ for Eq. (26). That is to say that we can use Eq. (13) to directly transform Eq. (26) to Eq. (28), and vice versa, without recourse to the formulation of Eq. (16) [i.e. an alternative form of Eq. (8) with its variable, $w$, being expressed as $v^{-1}$ ], which can be solved for GVF profiles in the domains of $|w / \lambda|<1$ (i.e. $|\lambda v|>1$ ), thereby proving that we can obtain Eq. (28) directly from Eq. (26) rather than indirectly through Eq. (16), vice versa. If we accept Eq. (10) is a definition of GHF for $|z|>1$, as treated by the commercial Mathematica software (Wolfram, 1996), then one of Eqs. (26) and (28) is enough to represent the complete GHF-based solutions of Eq. (8) for GVF profiles in adverse channels, covering the physically possible domain of 0 $\leq \lambda v<\infty$. Table 1 shows the summary of the critical-depthbased dimensionless GVF profiles expressed by using the GHF on all types of slopes.

\section{Discussions}

\subsection{Plotting the GHF-based solutions with $\lambda$ as a parameter}

Equations (21) and (23), which span respectively the domains of $0 \leq \lambda v<1$ and $\lambda v>1$, are the GHF-based solutions of Eq. (3) for $y_{\mathrm{c}}$-based dimensionless GVF profiles in sustaining channels, while Eqs. (26) and (28), covering respectively the domains of $0 \leq \lambda v \leq 1$ and $\lambda v \geq 1$, are GHFbased solutions of Eq. (8) for $y_{\mathrm{c}}$-based dimensionless GVF profiles in adverse channels. As explained in the last section, one of Eqs. (26) and (28) is enough to represent the complete GHF-based solutions of Eq. (8) for GVF profiles in adverse channels, covering the whole domain of $0 \leq \lambda v<\infty$. Equation (26) is chosen herein. A plot of Eqs. (21), (23) and (26) on the $\left(x_{\#}, v\right)$-plane with $\lambda$ as a parameter may help gain an insight into the uniqueness and versatility of such GHF-based solutions. We thus use $M=3$ (i.e. a typical value corresponding to the wide channels) and $N=10 / 3$ (i.e. a typical value corresponding to the Manning formula) as an example to plot the $y_{\mathrm{c}}$-based solution curves on the $\left(x_{\#}\right.$, 


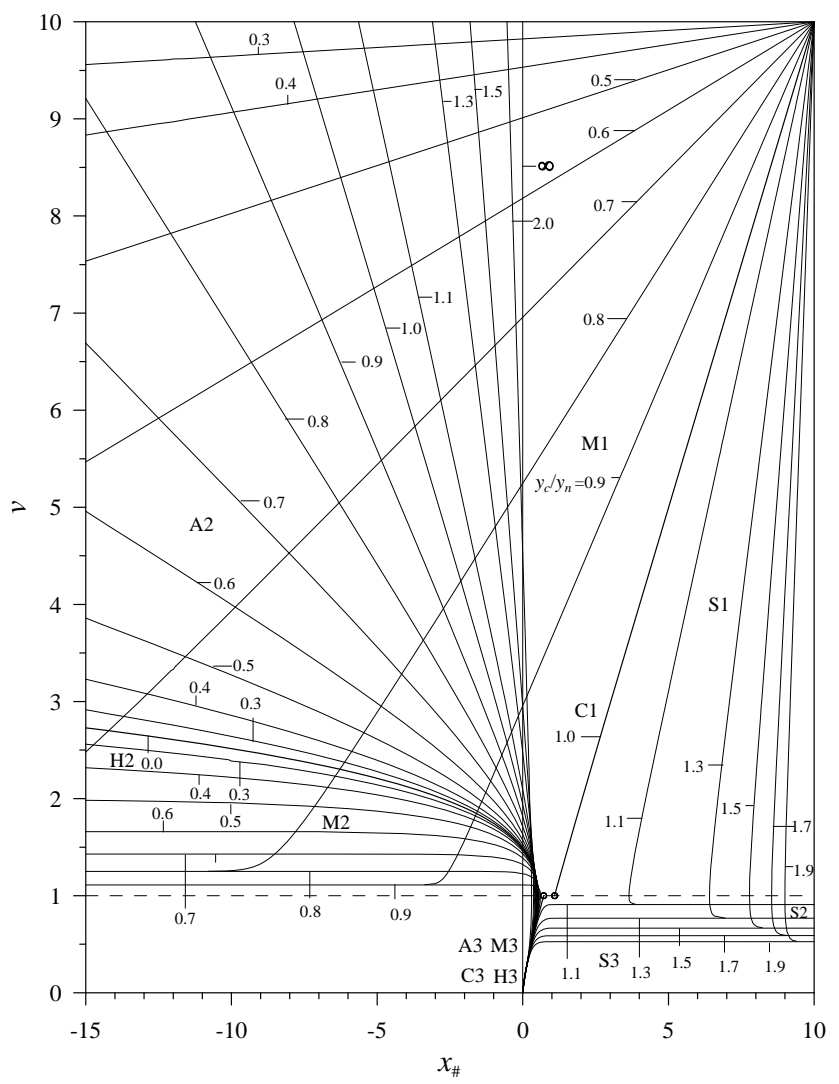

Fig. 1. A plot of the $y_{\mathrm{c}}$-based dimensionless 1-D GVF profiles for $M=3$ and $N=10 / 3$ with $y_{\mathrm{c}} / y_{\mathrm{n}}$ as a parameter.

$v$ )-plane herein, as shown in Fig. 1. In Fig. 1, three boundary conditions are arbitrarily prescribed at $\left(x_{\#}, v\right)=(10,10)$ for Eq. (23), and at $\left(x_{\#}, v\right)=(0,0)$ for Eqs. (20) and (26), respectively. There exist twelve types of the $y_{\mathrm{c}}$-based GVF profiles, two on the H slope $(\lambda=0)$, three on the $\mathrm{M}$ slope $(0<\lambda<1)$, two on the $\mathrm{C}$ slope $(\lambda=1)$, three on the $\mathrm{S}$ slope $(1<\lambda<\infty)$, and two on the A slope $(0 \leq \lambda<\infty$, in which $y_{\mathrm{n}}$ is fictitious). The twelve $y_{\mathrm{c}}$-based GVF profiles in three zones may be respectively referred to as $\mathrm{H} 2$ and $\mathrm{H} 3$; $\mathrm{M} 1$, $\mathrm{M} 2$, and M3; $\mathrm{C} 1$ and $\mathrm{C} 3 ; \mathrm{S} 1, \mathrm{~S} 2$, and S3; A2 and A3. The twelve profiles so classified are the same as those classified by Chow (1959) except for C2 (Chow treated it as for a uniform flow), which is excluded from our classification because it is just a singularity for all the $N$ values except for $N=3$, as treated by Jan and Chen (2012).

\subsection{Mild (M), steep (S), and adverse (A) profiles in zones 1,2 , and 3}

Examination of Fig. 1 reveals that two solution curves drawn for the critical value of $\lambda$ (i.e. $\lambda=y_{\mathrm{c}} / y_{\mathrm{n}}=1$ ) and the asymptotic value of $\lambda$ (i.e. $\lambda=0$ ) combined with the horizontal line at $v=1$ and the horizontal asymptotes at $\lambda v=1$ (or $\left.v=y_{\mathrm{n}} / y_{\mathrm{c}}\right)$ can divide the entire domain of the solution curves on the $\left(x_{\#}, v\right)$-plane into nine regions, which are constituted by a combination of three slopes and three zones, i.e. the same division adopted by Chow (1959). The three slopes consist of mild (M) slopes, steep (S) slopes, and adverse (A) slopes, while the three zones are composed of zone 1 ( $\lambda<v<\infty$ for M1 profiles or $1 \leq v<\infty$ for S1 profiles), zone 2 ( $1 \leq v<\lambda$ for M2 profiles, $\lambda<v \leq 1$ for $\mathrm{S} 2$ profiles, or $1 \leq v<\infty$ for A2 profiles), and zone $3(0 \leq v \leq 1$ for M3 profiles, $0 \leq v<\lambda$ for S3 profiles, or $0 \leq v \leq 1$ for $\mathrm{A} 3$ profiles). Apparently, the particular solution curves drawn by Eqs. (21) and (23) with $\lambda\left(=y_{\mathrm{c}} / y_{\mathrm{n}}\right)=1$ separate the region of $\mathrm{M}$ profiles from that of $\mathrm{S}$ profiles, while the unique solution curve constructed by Eq. (7) through the asymptotic reduction of Eq. (21) or (26) as $\lambda \rightarrow 0$ separate the region of $\mathrm{M}$ profiles from that of A profiles. These two solution curves are respectively referred to as the critical $(\mathrm{C})$ profiles on the critical $(\mathrm{C})$ slope $(\lambda=1)$ and the horizontal $(\mathrm{H})$ profiles on the horizontal $(\mathrm{H})$ slope $(\lambda=0)$.

\subsection{Critical (C) profiles in zones 1 and 3}

The particular solutions of Eqs. (21) and (23) on substitution of $\lambda=1$ yield

$$
\begin{aligned}
x_{\#}= & -\frac{v^{N+1}}{N+1} g\left(\frac{N+1}{N}, v^{N}\right)+\frac{v^{N-M+1}}{N-M+1} g \\
& \left(\frac{N-M+1}{N}, v^{N}\right)+\text { Const }
\end{aligned}
$$

$$
\begin{gathered}
x_{\#}=v g\left(-\frac{1}{N}, v^{-N}\right)+\frac{v^{-M+1}}{M-1} g \\
\left(\frac{M-1}{N}, v^{-N}\right)+\text { Const }
\end{gathered}
$$

which are specifically referred to as the equations for $y_{\mathrm{c}^{-}}$based $\mathrm{C} 3$ profiles in zone $3(0 \leq v<1)$ and $\mathrm{C} 1$ profiles in zone $1(1<v<\infty)$, respectively. In particular, upon substitution of $M=N=3$ and with the use of the recurrence formulas Eq. (12), Eq. (29) can readily reduce to

$x_{\#}=v+$ Const

which is a straight line. Likewise, we can prove that Eq. (30) on substitution of $M=N=3$ and with the help of the recurrence formula Eq. (12) also reduces to Eq. (31), a straight line. We can readily plot Eqs. (29) and (30) in Fig. 1 for all the values of $v$ except at $v=1$, where both equations are undefined due to the existence of a singularity. Two small open circles are used to mark the singularity in Fig. 1. It is selfevident from Fig. 1 that both equations so plotted for $\lambda=1$ irrespective of the existence of a singularity at $v=1$ are the lines of demarcation, which divide the domain of solutions curves obtained from Eqs. (21) and (23) into two regions, one for $\mathrm{M}$ profiles $(0<\lambda<1)$ and the other for $\mathrm{S}$ profiles $(\lambda>1)$. 


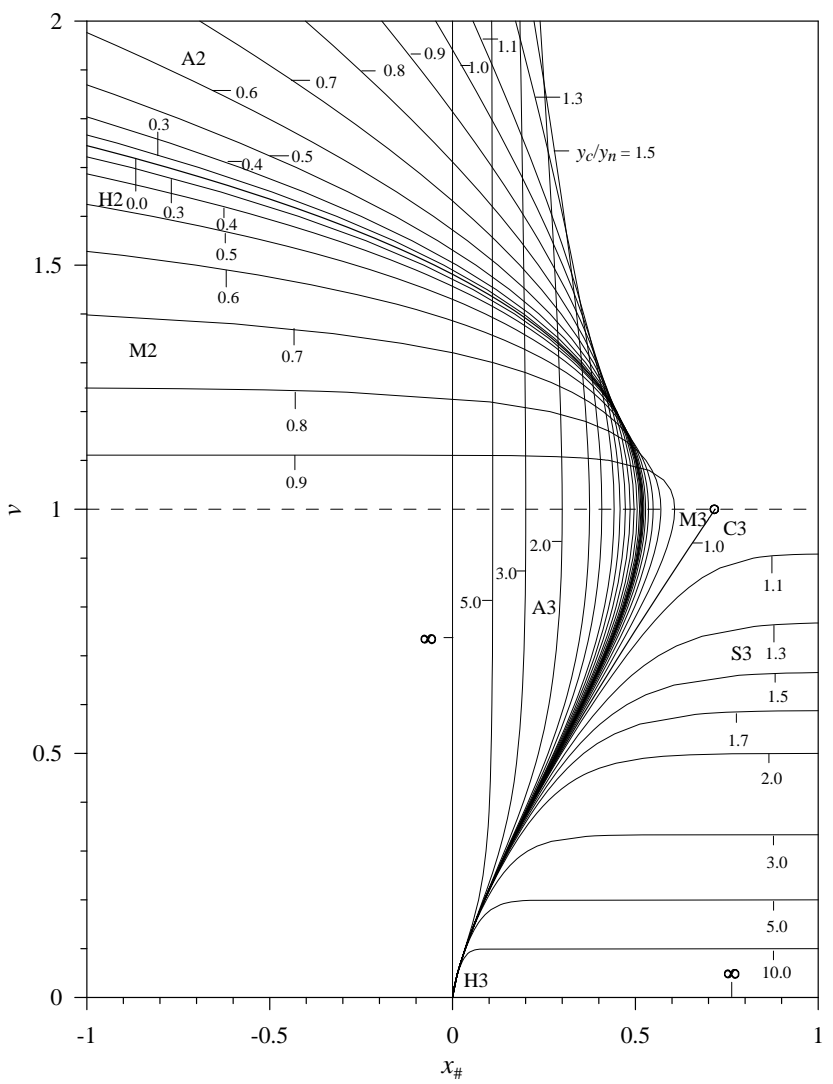

Fig. 2. A close-up of those $H, M, C, S$ and A profiles which are plotted in Fig. 1 around the boundary condition at $\left(x_{\#}, v\right)=(0,0)$.

\subsection{Horizontal $(\mathrm{H})$ profiles in zones 2 and 3}

To separate the region of $\mathrm{M}$ profiles from that of A profiles is a solution curve plotted by use of Eq. (7), to which Eqs. (21) and (26) reduce asymptotically as $\lambda \rightarrow 0$ from either side of Eq. (7), as shown in Fig. 1. For clarity in highlighting this line of demarcation, Eq. (7), i.e. a solution curve for $\lambda=0$, we draw Fig. 2, a close-up of Fig. 1 around the commonly prescribed boundary condition of Eqs. (7), (21) and (26) at ( $x_{\#}$, $v)=(0,0)$. One can easily prove that Eq. (7) can be obtained by the asymptotic reduction of Eq. (21) or (26) as $\lambda \rightarrow 0$, using the GHF definition. The A2 and A3 profiles plotted using Eq. (26) can also reduce asymptotically to Eq. (7) as $\lambda \rightarrow 0$. As displayed clearly in Fig. 2, the $\mathrm{H} 2$ profile divides perfectly between the regions of $\mathrm{M} 2$ profiles and $\mathrm{A} 2$ profiles; so does the $\mathrm{H} 3$ profile between the regions of $\mathrm{M} 3$ profiles and A3 profiles.

\subsection{Horizontal asymptotes at $v=y_{\mathrm{n}} / y_{\mathrm{c}}$ for various $\lambda$-values}

It is already known that Eqs. (21) and (23) diverge along the horizontal asymptotes. However, unlike the sole horizontal asymptote at $u=1$ on the $\left(x_{*}, u\right)$-plane to represent the water surface running parallel with the channel bed as $x_{*} \rightarrow \pm \infty$, as shown in the Fig. 1 of Jan and Chen (2012), the horizontal asymptotes at $v=\lambda^{-1}$ (i.e. $\lambda v=1$ ) for various $\lambda$-values on the $\left(x_{\#}, v\right)$-plane translate vertically, as shown in Fig. 1 herein. Accordingly, the horizontal asymptotes at $v=\lambda^{-1}$ on the $\left(x_{\#}, v\right)$-plane for the given $\lambda$-values may be deemed as "movable" lines of demarcation drawn to divide the domain of $v$ into two major regions: One region spans $0 \leq \lambda v<1$ (or $0 \leq v<\lambda^{-1}$ ), which covers both zones 2 and 3 for M profiles as well as zone 3 for $\mathrm{S}$ profiles, and the other region extends over $\lambda v>1$ (or $v>\lambda^{-1}$ ), which covers zone 1 for M profiles as well as both zones 1 and 2 for $\mathrm{S}$ profiles.

In comparison, there exist no horizontal asymptotes at $v=\lambda^{-1}$ for various $\lambda$-values in the domain of solution curves plotted for Eq. (26) on the $\left(x_{\#}, v\right)$-plane, which represent the $y_{\mathrm{c}}$-based dimensionless GVF profiles on the adverse slope. An inspection of Fig. 1 clearly reveals that the solution curves plotted for Eqs. (21) and (23) diverge at $v=\lambda^{-1}$ on the $\left(x_{\#}, v\right)$-plane, whereas those which are plotted for Eq. (26) do not diverge at $v=\lambda^{-1}$ for any $\lambda$-value shown. The latter result may be attributed to the fact that $y_{\mathrm{n}}$ adopted in the derivation of Eq. (26) is simply fictitious (i.e. nonexistent).

\subsection{Two inflection points of the $M$ profiles}

The inflection point of a flow profile on the $\mathrm{d} v / \mathrm{d} x_{\#}$ versus $v$ plane takes place at a point, where $\mathrm{d} v / \mathrm{d} x_{\#}$ is an extremum (i.e., either a minimum or a maximum). It lies either between the two vertical asymptotes or between the horizontal asymptote and its adjacent vertical asymptote. The v-value, at which the inflection point occurs, can be determined from the condition under which $d^{2} v / \mathrm{d} x_{\#}^{2}=0$. We can derive such a condition from the reciprocal of Eq. (3) as follows:

$\frac{d^{2} v}{\mathrm{~d} x_{\#}^{2}}=\frac{\left[1-\lambda^{N} v^{N}\right]\left[M \lambda^{N} v^{N}-N v^{M}+(N-M)\right]}{v^{2(N-M)+1}\left(1-v^{M}\right)^{3}}$.

Equating the right-hand side of the equal sign in Eq. (32) to zero yields

$M \lambda^{N} v^{N}-N v^{M}+(N-M)=0$.

For illustration, the two $y_{\mathrm{c}}$-based solutions of $v$ obtained from Eq. (33) for various $N$ values under each of the assumed $\lambda=0.6$ and 0.95 are tabulated in Table 2, where Eq. (33) for $N=10 / 3,17 / 5,7 / 2$, and $11 / 3$ are specifically referred to as Eqs. (33a-d), respectively. On the contrary, Eq. (33) can asymptotically reduce to the sole solution of $v$ at the inflection point of the $\mathrm{H} 3$ profile on the horizontal slope as $\lambda \rightarrow 0$, as shown in the following subsection. Eq. (33) is thus valid in the range of $0 \leq \lambda<1$, including one end point at $\lambda=0$ but excluding the other end point at $\lambda=1$.

In Fig. 3, we plot eight solution curves to trace the paths of the two inflection points of the M1 and M3 profiles on the $v$ versus $\lambda$ plane with $N$ as a parameter for all the $N$ values studied except $N=3$, thereby showing the combined effects of $\lambda$ and $N$ on the locations of the two inflection points. 
Table 1. The $y_{\mathrm{c}}$-based dimensionless GVF profiles expressed by using the GHF on all types of slopes except for the horizontal slope.

\begin{tabular}{|c|c|c|}
\hline $\begin{array}{l}\text { Types of GVF profiles } \\
\text { (valid domain of } v \text { ) }\end{array}$ & $\begin{array}{l}\text { Equations for dimensionless GVF profiles in } \\
\text { terms of GHF with hydraulic exponents, } M \text { and } N\end{array}$ & Eq. no. \\
\hline $\mathrm{H} 2(1 \leq v<\infty)$ & $x_{\#}=\frac{v^{N-M+1}}{N-M+1}-\frac{v^{N+1}}{N+1}+$ Const & (7) \\
\hline $\mathrm{H} 3(0 \leq v \leq 1)$ & $x_{\#}=\frac{v^{N-M+1}}{N-M+1}-\frac{v^{N+1}}{N+1}+$ Const & (7) \\
\hline $\begin{array}{ll}\text { M1 } & \left(\lambda^{-1}<v<\infty\right) \text { or } \\
& (1<\lambda v<\infty)\end{array}$ & $x_{\#}=\lambda^{-N} v g\left(-\frac{1}{N},(\lambda v)^{-N}\right)+\frac{\lambda^{-N} v^{-M+1}}{M-1} g\left(\frac{M-1}{N},(\lambda v)^{-N}\right)+$ Const & (23) \\
\hline $\begin{array}{ll}\mathrm{M} 2 & \left(1 \leq v<\lambda^{-1}\right) \text { or } \\
& (\lambda \leq \lambda v<1)\end{array}$ & $x_{\#}=-\frac{v^{N+1}}{N+1} g\left(\frac{N+1}{N},(\lambda v)^{N}\right)+\frac{v^{N-M+1}}{N-M+1} g\left(\frac{N-M+1}{N},(\lambda v)^{N}\right)+$ Const & (21) \\
\hline $\begin{array}{ll}\text { M3 } & (0 \leq v \leq 1) \text { or } \\
& (0 \leq \lambda v \leq \lambda)\end{array}$ & $x_{\#}=-\frac{v^{N+1}}{N+1} g\left(\frac{N+1}{N},(\lambda v)^{N}\right)+\frac{v^{N-M+1}}{N-M+1} g\left(\frac{N-M+1}{N},(\lambda v)^{N}\right)+$ Const & (21) \\
\hline $\mathrm{C} 1 \begin{array}{l}(1<v<\infty) \text { or } \\
(1<\lambda v<\infty)\end{array}$ & $x_{\#}=v g\left(-\frac{1}{N}, v^{-N}\right)+\frac{v^{-M+1}}{M-1} g\left(\frac{M-1}{N}, v^{-N}\right)+$ Const & (30) \\
\hline $\begin{array}{ll} & (0 \leq v<1) \text { or } \\
& (0 \leq \lambda v<1)\end{array}$ & $x_{\#}=-\frac{v^{N+1}}{N+1} g\left(\frac{N+1}{N}, v^{N}\right)+\frac{v^{N-M+1}}{N-M+1} g\left(\frac{N-M+1}{N}, v^{N}\right)+$ Const & (29) \\
\hline $\begin{array}{ll} & (1 \leq v<\infty) \text { or } \\
& (\lambda \leq \lambda v<\infty)\end{array}$ & $x_{\#}=\lambda^{-N} v g\left(-\frac{1}{N},(\lambda v)^{-N}\right)+\frac{\lambda^{-N} v^{-M+1}}{M-1} g\left(\frac{M-1}{N},(\lambda v)^{-N}\right)+$ Const & (23) \\
\hline $\begin{array}{ll} & \left(\lambda^{-1}<v \leq 1\right) \text { or } \\
& (1<\lambda v \leq \lambda)\end{array}$ & $x_{\#}=\lambda^{-N} v g\left(-\frac{1}{N},(\lambda v)^{-N}\right)+\frac{\lambda^{-N} v^{-M+1}}{M-1} g\left(\frac{M-1}{N},(\lambda v)^{-N}\right)+$ Const & (23) \\
\hline 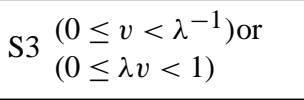 & $x_{\#}=-\frac{v^{N+1}}{N+1} g\left(\frac{N+1}{N},(\lambda v)^{N}\right)+\frac{v^{N-M+1}}{N-M+1} g\left(\frac{N-M+1}{N},(\lambda v)^{N}\right)+$ Const & (21) \\
\hline $\mathrm{A} 2 \begin{array}{l}(1 \leq v<\infty) \text { or } \\
(\lambda \leq \lambda v<\infty)^{\mathrm{a}}\end{array}$ & $x_{\#}=-\lambda^{-N} v g\left(-\frac{1}{N},-(\lambda v)^{-N}\right)-\frac{\lambda^{-N} v^{-M+1}}{M-1} g\left(\frac{M-1}{N},-(\lambda v)^{-N}\right)+$ Const & (28) \\
\hline $\begin{array}{ll} & (0 \leq v \leq 1) \text { or } \\
& (0 \leq \lambda v \leq \lambda)^{\mathrm{a}}\end{array}$ & $x_{\#}=-\frac{v^{N+1}}{N+1} g\left(\frac{N+1}{N},-(\lambda v)^{N}\right)+\frac{v^{N-M+1}}{N-M+1} g\left(\frac{N-M+1}{N},-(\lambda v)^{N}\right)+$ Const & (26) \\
\hline
\end{tabular}

${ }^{\text {a }}$ Here $\lambda=y_{\mathrm{c}} / y_{\mathrm{n}}$, for flow in a adverse channel, $y_{\mathrm{n}}$ is not real. It is presumed to be of a "fictitious" value obtainable from the same flow discharge in a "fictitious" channel on an identically sustaining (but negative) slope.

Table 2. The $y_{\mathrm{c}}$-based dimensionless flow depths at the two inflection points (IPs), one on the M1 profile and the other on the M3 profile, computed from Eq. (33) for the four $N$ values of GVF profiles on two mild slopes $\left(y_{\mathrm{c}} / y_{\mathrm{n}}=0.6\right.$ and 0.95$)$ in wide channels $(M=3)$.

\begin{tabular}{lccccc}
\hline & \multicolumn{2}{c}{$\lambda=y_{\mathrm{c}} / y_{\mathrm{n}}=0.6$} & \multicolumn{2}{c}{$\lambda=y_{\mathrm{c}} / y_{\mathrm{n}}=0.95$} \\
\cline { 2 - 6 } $\begin{array}{l}\text { Hydraulic } \\
\text { exponents } \\
(M, N)\end{array}$ & $\begin{array}{c}\text { M at IP on } \\
\left(\lambda^{-1}<v<\infty\right)\end{array}$ & $\begin{array}{c}v \text { at IP on } \\
(0 \leq v \leq 1)\end{array}$ & $\begin{array}{c}v \text { at IP on } \\
\text { M1 profile } \\
\left(\lambda^{-1}<v<\infty\right)\end{array}$ & $\begin{array}{c}\text { M at IP on } \\
(0 \leq v \leq 1)\end{array}$ & $\begin{array}{c}\text { Given the values of } \lambda, M \text {, and } N \\
\text { to solve Eq. }(33) \text { or }\end{array}$ \\
\hline$(3,10 / 3)$ & 226.861 & 0.4860 & 2.2296 & 0.6668 & $3 \lambda^{10 / 3} v^{10 / 3}-\frac{10}{3} v^{3}+\frac{1}{3}=0$ \\
\hline$(3,17 / 5)$ & 105.101 & 0.5111 & 2.0424 & 0.6875 & $3 \lambda^{17 / 5} v^{17 / 5}-\frac{17}{5} v^{3}+\frac{2}{5}=0$ \\
\hline$(3,7 / 2)$ & 48.622 & 0.5426 & 1.8640 & 0.7122 & $3 \lambda^{7 / 2} v^{7 / 2}-\frac{7}{2} v^{3}+\frac{1}{2}=0$ \\
\hline$(3,11 / 3)$ & 22.433 & 0.5842 & 1.6916 & 0.7425 & $3 \lambda^{11 / 3} v^{11 / 3}-\frac{11}{3} v^{3}+\frac{2}{3}=0$ \\
\hline
\end{tabular}




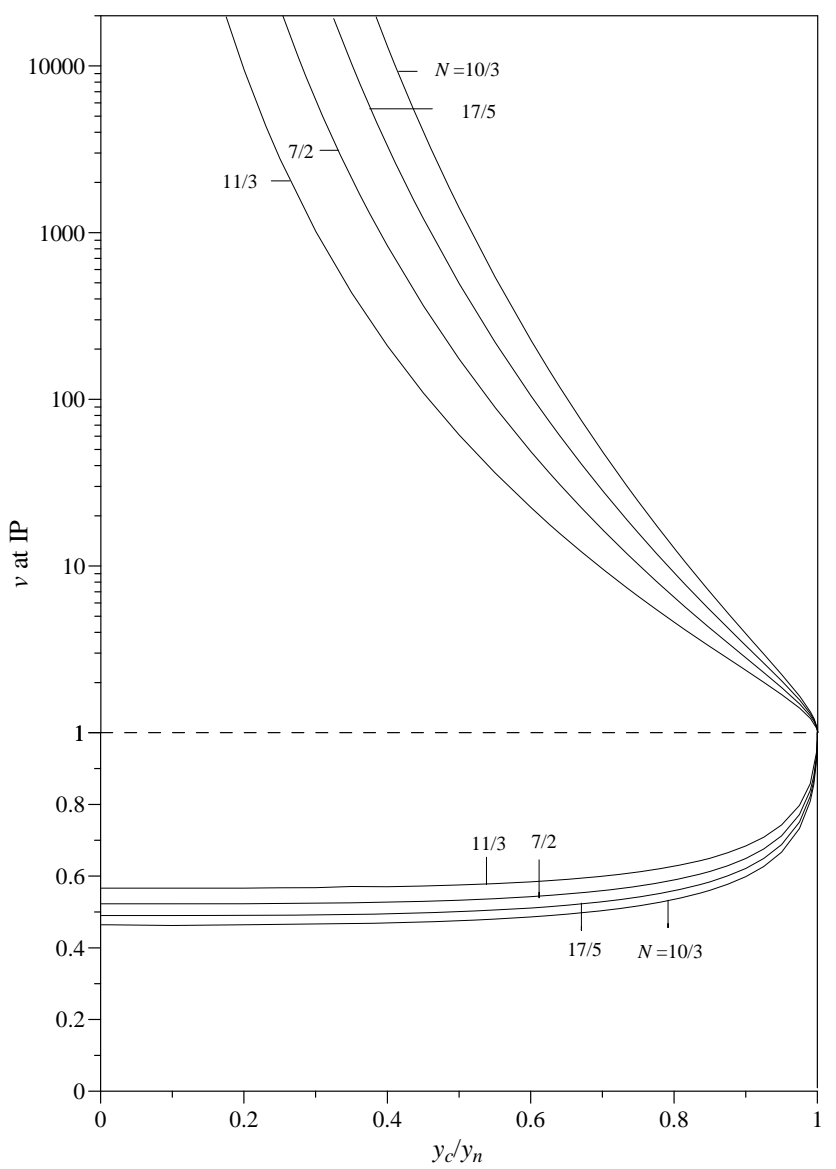

Fig. 3. Plot of eight solution curves representing the two values of $v$ at the inflection points (IP), one on each of the M1 and M3 profiles, against the various values of $y_{\mathrm{c}} / y_{\mathrm{n}}\left(0<y_{\mathrm{c}} / y_{\mathrm{n}} 1\right)$ under four $N$ values.

It merits mentioning that one of the distinctive advantages of the $y_{\mathrm{c}}$-based dimensionless GVF equation, Eq. (3), over the corresponding $y_{\mathrm{n}}$-based dimensionless GVF equation, Eq. (2), is reflected in Fig. 3 in which Eq. (33) can asymptotically reduce to the sole solution of $v$ at the inflection point of the $\mathrm{H} 3$ profile as $\lambda \rightarrow 0$. Brought up in the following for further discussion is the asymptotic reduction of Eq. (33) to the inflection point of the $\mathrm{H} 3$ profile as $\lambda \rightarrow 0$.

\subsection{Sole Inflection point of the $\mathrm{H} 3$ profile}

Given the values of $\lambda$ and $N$, we can use Eq. (33) to find the two inflection points of an $y_{\mathrm{c}}$-based dimensionless M profile, as pointed out above. We cannot only use Eq. (7) to plot the $y_{\mathrm{c}}$-based dimensionless $\mathrm{H}$ profiles for all the $N$ values studied, but also use (33) to determine the sole inflection point of an $y_{\mathrm{c}}$-based dimensionless $\mathrm{H} 3$ profile. To locate the sole inflection point is straightforward, i.e. Eq. (33) on substitution of $\lambda=0$ yields

$v=\left(\frac{N-M}{N}\right)^{1 / M}$ which is valid for all the $N$ values studied except $N=3$ in wide channels $(M=3)$. For H3 profiles with $N=10 / 3$, $17 / 5,7 / 2$, and $11 / 3$, the v-values at the inflection points for all such $N$ values can be computed from Eq. (34) to be $0.46416,0.49000,0.52276$, and 0.56652 , respectively, which should manifest themselves in Fig. 3. It is noted that Chen and Wang (1969) obtained the v-value at the sole inflection point equal to 0.464 for $N=10 / 3$, thus confirming the result computed from Eq. (34).

\subsection{Sole Inflection point of the A3 profile}

Using Eq. (8), we can determine the v-value, at which the inflection point of a GVF profile on the adverse slope takes place, from the condition under which $d^{2} v / \mathrm{d} x_{\#}^{2}=0$ as follows:

$\frac{d^{2} v}{\mathrm{~d} x_{\#}^{2}}=\frac{\left[1+\lambda^{N} v^{N}\right]\left[M \lambda^{N} v^{N}+N v^{M}-(N-M)\right]}{v^{2(N-M)+1}\left(1-v^{M}\right)^{3}}$.

Equating the right-hand side of the equal sign in Eq. (35) to zero yields

$M \lambda^{N} v^{N}+N v^{M}-(N-M)=0$,

because $y_{\mathrm{n}}$ so determined for GVF on the adverse slope is fictitious and so is the parameter, $\lambda\left[=\left(y_{\mathrm{c}} / y_{\mathrm{n}}\right)\right]$, defined in Eqs. (35) and (36), we should not mix them up with those defined in Eqs. (32) and (33), respectively. In contrast to the two solutions of $v$ which we have obtained from Eq. (33) for the given valid value of $\lambda$ and each of the $N$ values studied except $N=3$ in wide sustaining channels $(M=3)$, we can only find a sole solution of $v$ in Eq. (36), i.e. the condition for the existence of the inflection point for GVF profiles on the adverse slope. The numerical solutions of $v$ obtained from Eq. (36) for various values of $\lambda$ and $N$ reveal that Eq. (36) is valid for $0 \leq \lambda<\infty$, including one end point at $\lambda=0$, i.e. (34), to which (36) reduces asymptotically.

To gain an insight into the variation of the inflection point of the A3 profile on the $v$ versus $\lambda$ plane, we plot four solution curves with $N$ as a parameter, as shown in Fig. 4, each curve representing one of the four $N$ values studied, thereby showing the combined effects of $\lambda$ and $N$ on the path of the inflection point in the domain of $v$ against $\lambda$. The theoretical range of $\lambda$ is $0 \leq \lambda<\infty$, but for simplicity it is only plotted for $0 \leq \lambda<2$ in the figure due mainly to the space limit imposed on drawing the curves. In particular, at $\lambda=0$, Eq. (36) reduces to Eq. (34), which yields the unique v-value at the inflection point of an $\mathrm{H} 3$ profile for the given $N$ value in wide channels $(M=3)$. In a way, Eq. (34) plays the role of an interface between Eqs. (33) and (36) to switch the location of the inflection point from an $\mathrm{M} 3$ profile to an $\mathrm{A} 3$ profile, or vice versa, as $\lambda \rightarrow 0$. In fact, the role of Eq. (34) is analogous to that of Eq. (7), which intermediates between Eqs. (21) and (26) to switch GVF profiles from the M2 and M3 profiles to the $\mathrm{A} 2$ and $\mathrm{A} 3$ profiles, respectively, or vice versa, as $\lambda \rightarrow 0$. 


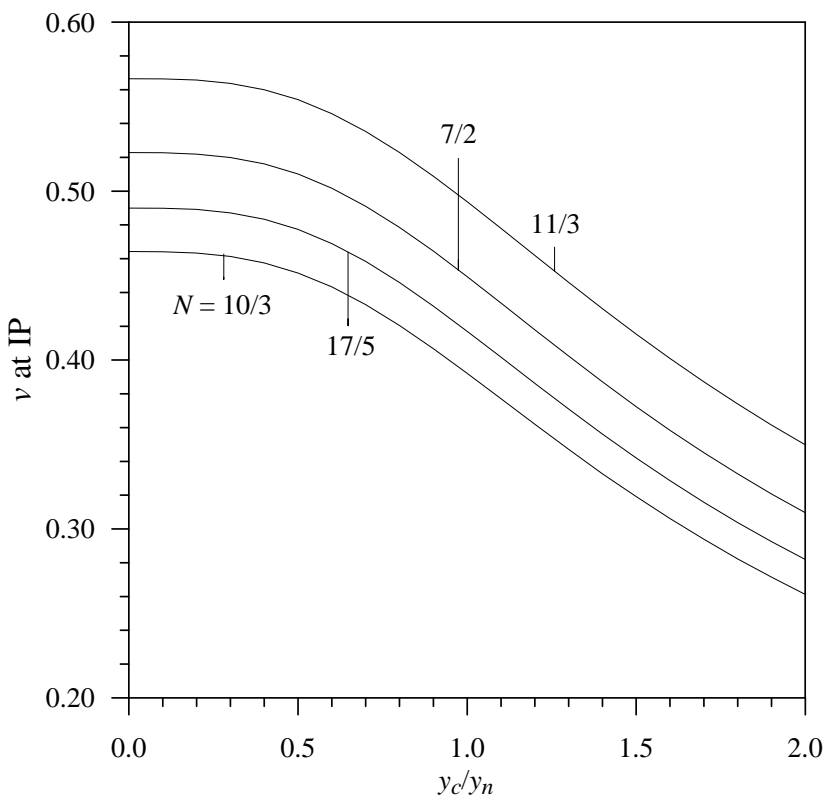

Fig. 4. Plot of four solution curves, each representing the single value of $v$ at the inflection point (IP) of the A3 profile against the various values of $y_{\mathrm{c}} / y_{\mathrm{n}}$ (in which $y_{\mathrm{n}}$ is fictitious) under four $N$ values.

\subsection{Curvature of the $y_{\mathrm{c}}$-based dimensionless GVF profiles}

The curvature, $K_{v}$, of the $y_{\mathrm{c}}$-based dimensionless GVF profiles at any point $\left(x_{\#}, v\right)$ can be expressed from calculus as

$K_{v}=\frac{\left|d^{2} v / \mathrm{d} x_{\#}^{2}\right|}{\left[1+\left(\mathrm{d} v / \mathrm{d} x_{\#}\right)^{2}\right]^{3 / 2}}$.

According to Eq. (37), the equations of $K_{v}$ for the $y_{\mathrm{c}}$-based dimensionless GVF profiles in sustaining channels and adverse channels can be expressed as Eqs. (38) and (39), respectively.

$$
\begin{aligned}
& K_{v}=\frac{\left|\left(1-\lambda^{N} v^{N}\right)\left[M \lambda^{N} v^{N}-N v^{M}+(N-M)\right]\right|}{\left|v^{-N+M+1}\right|\left\{\left[v^{N-M}\left(1-v^{M}\right)\right]^{2}+\left(1-\lambda^{N} v^{N}\right)^{2}\right\}^{3 / 2}} \\
& K_{v}=\frac{\left|\left(1+\lambda^{N} v^{N}\right)\left[M \lambda^{N} v^{N}+N v^{M}-(N-M)\right]\right|}{\left|v^{-N+M+1}\right|\left\{\left[v^{N-M}\left(1-v^{M}\right)\right]^{2}+\left(1+\lambda^{N} v^{N}\right)^{2}\right\}^{3 / 2}}
\end{aligned}
$$

Evidently, Eqs. (38) and (39) show that $K_{v}$ is zero at the inflection point by virtue of Eqs. (33) and (36), respectively; and so is at the place where the GVF profile becomes parallel with the bed at $y=y_{\text {n }}$ (i.e. $v=\lambda^{-1}$ ) in sustaining channels as a result of the zero factor in the numerator of Eq. (38), but not in adverse channels owing to the nonzero factor in the numerator of Eq. (39). For gaining an insight into the effects of $\lambda\left(=y_{\mathrm{c}} / y_{\mathrm{n}}\right)$ and $N$ on $K_{v}$ at $v=1$ at a glance, we use Eqs. (38) and (39) to plot $K_{v}$ at $v=1$ against $\lambda$ with $M=3$

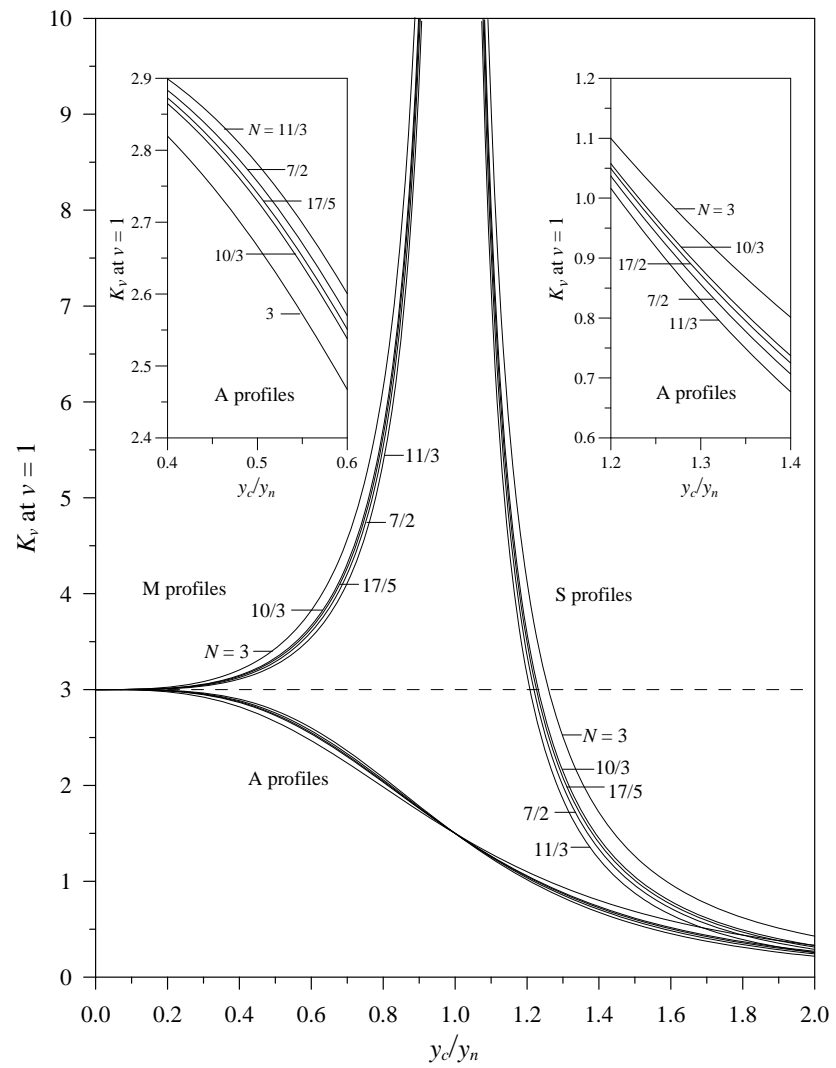

Fig. 5. Plot of the curvature, $K_{v}$, at $v=1$ against $y_{\mathrm{c}} / y_{\mathrm{n}}$ with the $N$ value as a parameter for GVF profiles in sustaining channels and also in adverse channels.

and $N$ value as a parameter, as shown in Fig. 5. The theoretical range of $\lambda$ in this plot is $0 \leq \lambda<\infty$, but it is only plotted for $0 \leq \lambda<2$ in the figure. An inspection of Fig. 5 reveals that $K_{v}$ at $v=1$ for GVF profiles in sustaining channels approaches infinity as $\lambda \rightarrow 1$. This trend for $K_{v}$ at $v=1$ implies that the closer the two $v$-values at the two inflection points of the $\mathrm{M}$ profiles approach unity, as shown in Fig. 3, the larger is $K_{v}$ at $v=1$, irrespective of the $\mathrm{M}$ or $\mathrm{S}$ profile, as shown in Fig. 5. In contrast, $K_{v}$ at $v=1$ for A profiles in adverse channels decreases and approaches zero as $\lambda \rightarrow \infty$. The A profile corresponding to this limit $(\lambda=\infty)$ is a vertical line with its $K_{v}$ everywhere along the line is zero, as manifesting itself in Fig. 1. As for the effect of $N$ on $K_{v}$ at $v=1$, Fig. 5 also shows that the smaller the $N$ value, the larger is $K_{v}$ at $v=1$ for all $\mathrm{M}, \mathrm{S}$, and A profiles in the whole range of $\lambda$, except that it tends to behave opposite for A profiles spanning $0 \leq \lambda \leq 1$.

\subsection{Applicability of the $y_{\mathrm{c}}$-based dimensionless GVF profiles}

The fact that $y_{\mathrm{c}}$-based dimensionless GVF profiles obtained herein by using GHF lies in its capability to reduce the $y_{\mathrm{c}}$-based $\mathrm{M}$ or A profiles asymptotically to the $y_{\mathrm{c}}$-based $\mathrm{H}$ 
profiles as $y_{\mathrm{c}} / y_{\mathrm{n}} \rightarrow 0$ has laid the foundation to compute at one sweep the $y_{\mathrm{c}}$-based dimensionless GVF profiles in a series of sustaining and adverse channels, which have horizontal slopes sandwiched in between them. Acceptance of any real numbers by the function parameters (i.e. $M$ and $N$ ) of the GHF suggests the practicality of the GHF-based solutions to the GVF profiles in channels with cross-sectional shape other than wide rectangle. For applying the GHF-based solutions obtained from Eqs. (3) and (8) to practical problems, our follow-up task needed to be undertaken is the formulation of various boundary conditions, which will be incorporated with the GHF-based solutions to compute at one sweep the $y_{\mathrm{c}}$-based dimensionless GVF profiles in a series of channels. The accurate formulation of various boundary conditions holds the key of success to compute the $y_{\mathrm{c}}$-based dimensionless GVF profiles in such a series of channels, though it is perhaps the hardest to develop a reliable method to conduct the computation constrained by such a variety of the boundary conditions.

As for types of the boundary conditions required for computing the $y_{\mathrm{c}}$-based dimensionless GVF profiles in a series of sustaining and adverse channels, we must first locate the internal boundary conditions, which exist at places where the state of flow suddenly changes (Chen and Wang, 1969; Chen and Chow, 1971). One type of the internal boundary conditions needed is at hydraulic jumps and overfalls, which occur in prismatic channels at places where the flow condition changes rapidly from a supercritical state to a subcritical state and vice versa under "freely" flowing conditions. The other type of the internal boundary conditions needed is at sudden or rapid transitions in channel width and crosssectional shape under "forced" flowing conditions as a result of constricted flows passing through hydraulic structures, such as weirs and sluice gates built in non-prismatic channels. Apparently, there exist various types of the internal boundary conditions, such as the hydraulic-jump equations at places where hydraulic jumps occur, the formation of the critical Froude number at places where overfalls are induced, and the calibrated discharge relations (or rating curves) at places where weirs and sluice gates among other dischargemeasuring devices are installed. In fact, it is quite involved to compute the $y_{\mathrm{c}}$-based dimensionless GVF profiles subject to such a variety of the internal boundary conditions imposed at many places as needed in a series of artificially or naturally formed prismatic and non-prismatic channels. It is indeed challenging to undertake such computation though it is beyond the scope of this paper.

\subsection{Comparison of GVF profile obtained by the present method and that by the fourth order Runge-Kutta method}

Solving the GVF profile by using a fourth order Runge-Kutta method is in the field of numerical method. The result from the numerical method cannot provide total length of the wa- ter surface profile with a single computation. The present method can obtain an analytical solution, so it can obtain the water depth at a specified location in a single computation. The computation of Gaussian hypergeometric function is well established in commercial software, such as Matlab and Mathematica. No more programming effort is needed by using the Gaussian hypergeometric function. A comparison of the result obtained by the present method and that by the fourth order Runge-Kutta method is presented in the Supplement. For comparison, we take the Example 5.8 in the book of K. Subramanya (2009), with title Flow In Open Channels. The numerical code written for the commercial software Matlab by using the present method, the numerical code for Matlab by using the standard fourth order Runge-Kutta method, and the comparison of M1-profiles obtained by the present method and fourth order Runge-Kutta method are all shown in the Supplement. It is less $10 \mathrm{~s}$ for the computations of this example by these two methods. The numerical error in the water depth obtained by the standard fourth order Runge-Kutta method is about $2 \%$ at the longitudinal coordinate $\mathrm{x}=-8 \mathrm{~km}$.

In addition, it should be noted that the assumption of constant hydraulic exponents ( $M$ and $N$ ) has been made in the direct integration method to solve GVF profiles. Therefore, a suitable choice of representative hydraulic exponents for a concerned channel length is important. Even though, the assumption of constant hydraulic exponents is satisfactory in most rectangular and trapezoidal channels, the hydraulic exponents may vary appreciably with respect to the depth of flow when the channel section has abrupt changes in crosssectional geometry or is topped with a gradually closing crown. In such cases, the channel length should be divided into a number of reaches in each of which the hydraulic exponents appear to be constant (Chow, 1959, p. 260).

\section{Conclusions}

Success to formulate the normal-depth $\left(y_{\mathrm{n}}\right)$-based GVF profiles expressed in terms of GHFs for flow in sustaining channels, as reported by Jan and Chen (2012), does not warrant that it can likewise prevail to use $y_{\mathrm{n}}$ in the normalization of the GVF equation for flow in horizontal and adverse channels because $y_{\mathrm{n}}$ for an assumed uniform flow in horizontal and adverse channels is undefined. This paper has laid the foundation to compute at one sweep the critical-depth $\left(y_{\mathrm{c}}\right)$-based GVF profiles in a series of sustaining and adverse channels, which have horizontal slopes sandwiched in between them. To obtain the GHF-based dimensionless solutions from the $y_{\mathrm{c}}$-based GVF equation is our first step for developing a viable method to compute the $y_{\mathrm{c}}$-based dimensionless GVF profiles subject to a variety of the boundary conditions imposed in such a series of interconnected sustaining and adverse channels. Working toward that goal, we have come up with two significant results produced from this study: Firstly, 
we have obtained the GHF-based solutions from the $y_{\mathrm{c}}$-based dimensionless GVF equation, which proves to be applicable for computing the GVF profiles in both sustaining and adverse channels. Secondly, we have analytically proved that the GHF-based dimensionless $\mathrm{M}$ and A profiles, if normalized by $y_{\mathrm{c}}$ rather than by $y_{\mathrm{n}}$, can asymptotically reduce to the $y_{\mathrm{c}}$-based dimensionless $\mathrm{H}$ profiles as $y_{\mathrm{c}} / y_{\mathrm{n}} \rightarrow 0$. Both significant results thus constitute the principal conclusions drawn from this study.

In practical applications, the $y_{\mathrm{c}}$-based dimensionless GVF profiles expressed in terms of the GHF can prove to be more useful, versatile than the $y_{\mathrm{n}}$-based equivalents obtained by Jan and Chen (2012) though both profiles are convertible to each other through the scaling relations, Eqs. (4) and (5). Among the well-known advantages of such $y_{\mathrm{c}}$-based dimensionless GVF profiles over their counterparts based on $y_{\mathrm{n}}$, there lies the most powerful feature of the $y_{\mathrm{c}}$-based GVF profiles expressed in terms of the GHF, with which one can readily reduce the $y_{\mathrm{c}}$-based $\mathrm{M}$ and A profiles asymptotically to the $y_{\mathrm{c}}$-based $\mathrm{H}$ profiles as $y_{\mathrm{c}} / y_{\mathrm{n}} \rightarrow 0$. In fact, we have proved that the M2 and M3 profiles can asymptotically reduce to the $\mathrm{H} 2$ and $\mathrm{H} 3$ profiles, respectively, as $y_{\mathrm{c}} / y_{\mathrm{n}} \rightarrow 0$; and so can the $\mathrm{A} 2$ and $\mathrm{A} 3$ profiles to the $\mathrm{H} 2$ and $\mathrm{H} 3$ profiles, respectively.

After decades-long struggle by hydraulicians in their attempts to improve the rudimentary approach taken to solve the GVF equation using the direct integration method, based on $y_{\mathrm{n}}$ and the varied-flow function, we have finally come up with a novel approach to solve the same problem based on $y_{\mathrm{c}}$ and the GHF instead. As shown in Fig. 1, an innovated formulation of the $y_{\mathrm{c}}$-based dimensionless GVF profiles expressed in terms of the GHFs has greatly advanced the conventional technique used in the GVF computation to the extent that hydraulicians for the first time in the computer age can fully utilize a mathematics software, which is capable of producing the GHF-based solutions of the $y_{\mathrm{c}}$-based dimensionless GVF equation. The principal conclusions so drawn from this study embrace all significant results acquired from the in-depth analysis of the $y_{\mathrm{c}}$-based dimensionless solutions expressed in terms of the GHF along with those attained in the exact proof of the asymptotic reduction of the $y_{\mathrm{c}}{ }^{-}$ based dimensionless $\mathrm{M}$ and A profiles to the corresponding $\mathrm{H}$ profiles as $y_{\mathrm{c}} / y_{\mathrm{n}} \rightarrow 0$.

\section{Supplementary material related to this article is available online at: http://www.hydrol-earth-syst-sci.net/ 17/973/2013/hess-17-973-2013-supplement.pdf.}

Acknowledgements. Support from the National Science Council in Taiwan (NSC 100-2221-E-006-202-MY3) to C. D. Jan for this study is acknowledged. The help from W. L. Ke in preparing the supplement is also acknowledged. C. L. Chen was born in 1931 and passed away on 25 January 2012. He was used to be an excellent hydrologist in US Geological Survey, and a distinguished professor in University of Illinois at Urbana-Champaign and in Utah State University.

Edited by: E. Todini

\section{References}

Allen, J. and Enever, K. J.: Water surface profiles in gradually varied open-channel flow, Proceedings, Institute of Civil Engineers, 41, 783-811, 1968.

Bakhmeteff, B. A.: Hydraulics of Open Channels. McGraw-Hill, New York, N.Y., 1932

Chen, C. L.: Unified theory on power laws for flow resistance, J. Hydraul. Eng., ASCE, 117, 371-389, 1991.

Chen, C. L. and Chow, V. T.: Formulation of mathematical watershed-flow model, J. Eng. Mech. Divis., ASCE, 97, 809828, 1971.

Chen, C. L. and Wang, C. T.: Nondimensional gradually varied flow profiles, J. Hydraul. Divis., ASCE, 95, 1671-1686, 1969.

Chow, V. T.: Integrating the equation of gradually varied flow. Proceedings Paper No. 838, ASCE, 81, 1-32, 1955.

Chow, V. T.: Closure of Discussions on Integrating the equation of gradually varied flow, J. Hydraul. Divis., ASCE, 83, 9-22, 1957.

Chow, V. T.: Open-Channel Hydraulics. McGraw-Hill, New York, N.Y., 1959.

Jan, C. D. and Chen, C. L.: Use of the Gaussian hypergeometric function to solve the equation of gradually-varied flow, J. Hydrol., 456-457, 139-145, 2012.

Korn, G. A. and Korn, T. M.: Mathematical Handbook for Scientists and Engineers. McGraw-Hill, New York, N.Y., 1961.

Kumar, A.: Integral solutions of the gradually varied equation for regular and triangular channels. Proceedings, Institute of Civil Engineers, 65, 509-515, 1978.

Kumar, A.: Gradually varied surface profiles in horizontal and adversely sloping channels. Proceedings, Institute of Civil Engineers, 67, 435-452, 1979.

Kummer, E. E.: Über die hypergeometrische Reihe, Journal für die reine und angewandte Mathematik, 15, 39-83 and 127-172, 1836.

Luke, Y. L.: Mathematical Functions and Their Approximations, Academic Press Inc., New York, N.Y., 1975.

Matzke, A. E.: Varied flow in open channels of adverse slope, Transactions, ASCE, 102, 651-660, 1937.

Olde Daalhuis, A. B.: Hypergeometric function, in: NIST Handbook of Mathematical Functions, edited by: Olver, F. W. J., Lozier, D. W., Boisvert, R. F., and Clark, C. W., Cambridge Univ. Press, Cambridge (Chapter 15), 2010.

Pearson, C. E. (Ed.): Handbook of Applied Mathematics, Selected Results and Methods. Van Nostrand Reinhold Company, New York, N.Y., 1974.

Rouse, H.: Critical analysis of open-channel resistance, J. Hydraul. Divis., ASCE, 91, 1-25, 1965.

Seaborn, J. B.: Hypergeometric Functions and Their Applications. Springer-Verlag, New York, N.Y., 1991.

Subramanya, K.: Flow in Open Channels, 3rd Edn. McGraw-Hill, Singapore, 2009.

Vatankhah, A. R.: Direction integration of manning-based GVF equation in trapezoidal channels, J. Hydrol. Eng., ASCE, 17, 
455-462, 2012.

Venutelli, M.: Direct integration of the equation of gradually varied flow. J. Irrig. Drain. Eng., ASCE, 130, 88-91, 2004.

Wolfram, S.: The Mathematica Book, third ed. Wolfram Media \& Cambridge University Press, Champaign, Illinois, 1996.
Zaghloul, N. A.: A computer model to calculate varied flow functions for circular channels, Adv. Eng. Softw., 12, 106-122, 1990.

Zaghloul, N. A.: Gradually varied flow in circular channels with variable roughness, Adv. Eng. Softw., 15, 33-42, 1992. 